\title{
Metal strips and wires as plasmonic waveguides for integrated-optics components
}

Boltasseva, Alexandra; Leosson, Kristjan; Bozhevolnyi, Sergey I.; Søndergaard, Thomas; Jørgensen, K.B.; Pedersen, R.H.; Kristensen, Anders

Published in:

Quantum Electronics and Laser Science Conference, 2007. QELS '07

Publication date:

2007

Document Version

Publisher's PDF, also known as Version of record

Link back to DTU Orbit

Citation $(A P A)$ :

Boltasseva, A., Leosson, K., Bozhevolnyi, S. I., Søndergaard, T., Jørgensen, K. B., Pedersen, R. H., \& Kristensen, A. (2007). Metal strips and wires as plasmonic waveguides for integrated-optics components. In Quantum Electronics and Laser Science Conference, 2007. QELS '07 (pp. 1-2). IEEE.

\section{General rights}

Copyright and moral rights for the publications made accessible in the public portal are retained by the authors and/or other copyright owners and it is a condition of accessing publications that users recognise and abide by the legal requirements associated with these rights.

- Users may download and print one copy of any publication from the public portal for the purpose of private study or research.

- You may not further distribute the material or use it for any profit-making activity or commercial gain

- You may freely distribute the URL identifying the publication in the public portal 


\title{
Metal Strips and Wires as Plasmonic Waveguides for Integrated-Optics Components
}

\author{
Alexandra Boltasseva \\ COM•DTU, Department of Communications, Optics \& Materials, Nano $\bullet$ DTU, Technical University of Denmark, Building 345v, \\ DK-2800 Kongens Lyngby, Denmark \\ aeb@com.dtu.dk \\ Kristjan Leosson \\ Science Institute, University of Iceland, Dunhaga 3, IS-107 Reykjavik, Iceland \\ Sergey I. Bozhevolnyi, Thomas Søndergaard \\ Department of Physics and Nanotechnology, Aalborg University, Skjernvej 4a, DK-9220 Aalborg Øst, Denmark
}

Kasper B. Jørgensen, Rasmus H. Pedersen, Anders Kristensen

MIC - Department of Micro and Nanotechnology, Nano $\bullet$ DTU, Technical University of Denmark

\begin{abstract}
Propagation of long-range surface plasmon polaritons in different waveguide components based on $\mathrm{nm}$-thin and $\mu \mathrm{m}$-wide metal strips and symmetrical sub-wavelength metal nanowires embedded in a uniform dielectric is experimentally studied at telecom wavelengths.

(C)2007 Optical Society of America

OCIS codes: (240.6680) Surface plasmons; (250.5300) Photonic integrated circuits
\end{abstract}

The recent development of information technology demands miniaturization and increased functionality in integrated optical circuits. One of the avenues in the field of highly integrated photonic devices that recently has attracted considerable interest is optics based on the controlled excitation of surface plasmon polaritons (SPPs) [1]. SPPs represent quasi-two-dimensional electromagnetic (EM) excitations propagating along a dielectric-metal interface having the EM field amplitudes strongly enhanced at the interface and decaying exponentially into both neighboring media [1]. Tight field intensity confinement to the metal surface (typically on the order of or smaller than the wavelength in the corresponding media) allows one to manipulate the SPP in the surface plane by using different types of surface metal nanostructures. With the recent progress in the fabrication of different metal nanostructures, efficient control of the SPP propagation was achieved in different scattering and guiding surface configurations [2,3]. However, when compared to conventional dielectric waveguides used in integrated optics, SPPs suffer from strong damping due to absorption in metals so that their propagation length is limited to micrometer distances $(\sim 30 \mu \mathrm{m}$ in visible and $\sim 300 \mu \mathrm{m}$ in near infrared, for a silver-air interface). Moreover, SPPs are difficult to excite by the conventional end-fire technique and more elaborate schemes (grating- or prismcoupling) have to be used.

These limitations to the applicability of SPP-based waveguiding structures led to explorations of a special SPP subset, namely long-range surface plasmon polaritons (LR-SPPs) [4] propagating on nanometer-thin metal films or strips embedded in a dielectric material. Propagation of LR-SPP modes was first studied by Sarid [4] for thin, infinitely wide metal films and later considered by Berini for metal films of finite width [5]. Such a symmetrical structure supports propagation of the field-symmetric modal solution, whereby a coupling of SPPs at two metaldielectric interfaces results in a very small field concentration within the central metal film and, hence, low propagation loss for the LR-SPP. At the same time, for sufficiently thin metal films, the LR-SPP field components extend over several micrometers into the cladding via two identical evanescent tails, facilitating the optical excitation using the end-fire technique [6,7]. For a strip waveguide embedded in dielectric, the dimensions of the metal strip can be chosen so that the LR-SPP field distribution matches that of a single-mode fiber, facilitating the efficient end-fire excitation with coupling loss of less than $1 \mathrm{~dB}$ per facet [8,9]. Efficient LR-SPP excitation and guiding (at telecom wavelengths) along thin gold strips embedded in a dielectric was realized demonstrating a coupling loss of $\sim 0.5 \mathrm{~dB}$ and propagation loss of $\sim 6-8 \mathrm{~dB} / \mathrm{cm}$ for BCB-Au-BCB configuration [8] and propagation loss as low as $4.2 \mathrm{~dB} / \mathrm{cm}$ combined with $98 \%$ excitation efficiency for polymer-Au-glass configuration [9].

Low propagation and coupling loss attainable with LR-SPPs stimulated experimental studies of LR-SPP-based integrated optics (IO), and different passive components including straight and bent waveguides, Y-splitters, multimode interference devices and directional couplers (DCs) were demonstrated [8,9]. As an alternative approach for making IO circuits, LR-SPP stripe waveguides have one unique feature - the possibility of using the same metal- 
stripe circuitry for both guiding optical radiation and transmitting electrical signals that control its propagation. Lately, efficient LR-SPP-based dynamic devices with low power consumption, including various modulators and switches, have been also realized utilizing the thermo-optic effect in the polymer cladding [10]. Together with different passive and active LR-SPP-based components for IO, two different approaches for making Bragg gratings based on LR-SPP-supporting configurations (by varying the width [11] or thickness [12] of the metal stripe) were developed demonstrating a very broad range of LR-SPP-based grating performance (from weak narrow-band gratings [11] up to very strong and broad-band gratings [12]).

However, applicability of metal strip waveguides to an optical network with arbitrary polarization state is limited due to the fact that LR-SPP waveguides only support propagation of TM polarized light. Possible solution to this problem is a symmetric metal nanowire embedded in a dielectric. Metal nanowire with close to symmetric (square) cross sections with sub-micrometer dimensions was proposed in a US patent application by Berini [13]. Such plasmonic waveguides can be used to guide both TE and TM polarized light with reasonable propagation and coupling losses as experimentally demonstrated for gold nanowires (150-nm square cross section) embedded in a polymer [14]. Recently, the applicability of such nanowire waveguide in a simple thermo-optical variable optical attenuator device was also demonstrated [14].

Experimental investigations of different waveguide components (including bends, couplers, Bragg gratings and two-dimensional photonic crystals) based on LR-SPP supporting metal strips demonstrate that such plasmonic waveguides constitute a promising alternative for IO circuits. For applications where polarization is random or changing, metal nanowire waveguides are shown to be suitable candidates with efficient guiding of both TE and TM polarized light. Giving the unique possibility of using the same metal strips for both guiding light and transmitting electrical signals, we believe that such plasmonic waveguides can be used for efficiently controlling signals in integrated optical circuits.

[1] H. Raether, Surface Plasmons (Berlin: Springer, 1988).

[2] W. L. Barnes, A. Dereux, and T. W. Ebbesen, "Surface plasmon subwavelength optics," Nature, vol. 424, pp. 824-830, 2003.

[3] H. Ditlbacher, J. R. Krenn, G. Schider, A. Leitner, and F. R. Aussenegg, "Two-dimensional optics with surface plasmon polaritons," Appl. Phys. Lett., vol. 81, pp. 1762-1764, 2002. S. I. Bozhevolnyi, J. Erland, K. Leosson, P. M. W. Skovgaard, and J. M. Hvam, "Waveguiding in surface plasmon polariton band gap structures," Phys. Rev. Lett., vol. 86, pp. 3008-3011, 2001.

[4] D. Sarid, "Long-range surface-plasma waves on very thin metal films," Phys. Rev. Lett., vol. 47, pp. 1927-1930, 1981.

[5] P. Berini, "Plasmon-polariton waves guided by thin lossy metal films of finite width: Bound modes of symmetric structures," Physical Review B, vol. 61, pp. 10484-10503, 2000.

[6] J. J. Burke, G. I. Stegeman, and T. Tamir, "Surface-polariton-like waves guided by thin, lossy metal films," Physical Review B, vol. 33, pp. 5186-5201, 1986.

[7] R. Charbonneau, P. Berini, E. Berolo, and E. Lisicka-Skrzek, "Experimental observation of plasmon-polariton waves supported by a thin metal film of finite width," Optics Letters, vol. 25, pp. 844-846, 2000.

[8] A. Boltasseva, T. Nikolajsen, K. Leosson, K. Kjaer, M. S. Larsen, and S. I. Bozhevolnyi, "Integrated optical components utilizing long-range surface plasmon polaritons," J. Lightwave. Technol., vol. 23, pp. 413-422, 2005.

[9] P. Berini, R. Charbonneau, N. Lahoud, and G. Mattiussi, "Characterization of long-range surface-plasmon-polariton waveguides," Journal of Applied Physics, vol. 98, pp. 043109-01-12, 2005.

[10] T. Nikolajsen, K. Leosson, and S. I. Bozhevolnyi, "In-line extinction modulator based on long-range surface plasmon polaritons," Optics Communications, vol. 244, p. 455, 2005.

[11] S. Jetté-Charbonneau, R. Charbonneau, N. Lahoud, G. Mattiussi, and P. Berini, "Demonstration of Bragg gratings based on long-ranging surface plasmon polariton waveguides," Optics Express, vol. 13, pp. 4672-4682, 2005.

[12] S. I. Bozhevolnyi, A. Boltasseva, T. Søndergaard, T. Nikolajsen, and K. Leosson, "Photonic band gap structures for long-range surface plasmon polaritons," Optics Communications, vol. 250, pp. 328-333, 2005.

[13] P. Berini, “Optical Waveguide Structures,” US patent number 6,741,782 (http://www.uspto.gov/).

[14] K. Leosson, T. Nikolajsen, A. Boltasseva, and S. I. Bozhevolnyi, "Long-range surface plasmon polariton nanowire waveguides for device applications," Optics Express 14(1), 314 (2006). 